\section{Assessment of Neuroprotective Effects of Neurotropin in Cultured Rat Dorsal Root Ganglion Neurons Using High- Throughput Imaging}

\section{Abstract}

Background: Neurotropin, a non-protein extract derived from inflamed rabbit skin following inoculation with vaccinia virus, is an analgesic agent. The mechanism of action of neurotropin has been partially clarified. No experimental study, however, has been undertaken to assess oxaliplatin-induced neurotoxicity using quantitative in vitro high-throughput image analysis. In the present study, to elucidate the action of neurotropin on dorsal root ganglion (DRG) neurons, we explored the antioxidative and anti-inflammatory activity of neurotropin. We assessed the viability and morphological changes in cultured rat DRG neurons treated with oxaliplatin and neurotropin.

Methods: We plated dissociated DRG neurons from female Sprague-Dawley rats (100-120 g) into 96-well plates and added oxaliplatin and/or neurotropin. The cells were examined with a fluorescence microscope, ImageXpress. The images were analyzed with high content image processing software, MetaXpress. Cells treated with neurotropin and oxaliplatin were lysed in Pierce RIPA Buffer and subjected to western blot analysis.

Results: Significant increases in neurite length, neurite area, cell body area, and neurite branching were observed in the presence of neurotropin in DRG neurons treated with oxaliplatin. When the highest concentration of neurotropin, 40 neurotropin units (NU) $\mathrm{mNU} / \mathrm{mL}$, was applied, a marked effect was seen. Neurotropin did not affect the phosphorylation of c-Jun N-terminal kinase, but suppressed the phosphorylation of $\mathrm{p} 38$. This finding suggests that neurotropin reversed oxaliplatin-induced apoptosis via suppression of the p38 mitogenactivated protein kinase signaling pathway.

Conclusion: The results of this in vitro study suggest a possible role for neurotropin as a useful neuroprotective and anti-apoptotic agent.

Keywords: Neurotropin; Oxaliplatin; Neurotoxicity; Dorsal root ganglion neuron; Apoptosis; High-throughput imaging
Satoshi Akita, Mitsunori Sato*, Motohira Yoshida, Kei Ishimaru, Shigehiro Koga, Yusuke Matsuno, Jun Kuwabara, Kazufumi Tanigawa and Yuji Watanabe

Department of Gastrointestinal Surgery and Surgical Oncology, Ehime University Graduate School of Medicine, Shitsukawa, Toon, Ehime, Japan

*Corresponding author: Mitsunori Sato

झ mit-sato@m.ehime-u.ac.jp

Department of Gastrointestinal Surgery and Surgical Oncology, Ehime University Graduate School of Medicine, Shitsukawa, Toon, Ehime 790-0295, Japan.

Tel: +81899605975

Fax: +81899605976

Citation: Akita S, Sato M, Yoshida M, Ishimaru K, Koga S, et al. (2017) Assessment of Neuroprotective Effects of Neurotropin in Cultured Rat Dorsal Root Ganglion Neurons Using High-Throughput Imaging. J Biomedical Sci. Vol.6 No.5:35

Received: November 28, 2017; Accepted: December 08, 2017; Published: December 18,2017

\section{Introduction}

Colorectal cancer, which is a major global public health problem, is the most commonly diagnosed cancer and the fourth cause of cancer death worldwide [1]. However, colorectal cancer death rates declined by about $3 \%$ per year for both males and females between 2003 and 2012 [2]. Oxaliplatin, a platinum-based chemotherapy drug, is effective in the treatment of advanced metastatic colorectal cancer. However, it causes serious acute neurotoxicity (triggered by cold exposure) and chronic peripheral neurotoxicity (degeneration of sensory and motor nerves) in patients. Peripheral neurotoxicity is the most frequent dose-limiting toxicity with oxaliplatin [3]. A previous study demonstrated that a large percentage of patients underwent 
dose reductions, which can significantly affect the efficacy of treatment [4].

Acute neurotoxicity, which is characterized by abnormal sensation, occurs at low cumulative doses within hours or days of therapy. Patients recover after several hours or days. Oxaliplatin-induced acute neurotoxicity may not be explained by morphological damage to the peripheral nerves [5]. Chronic neurotoxicity is related to the cumulative oxaliplatin dose and persists for several months. In contrast to acute neurotoxicity, the cumulative neurotoxicity due to oxaliplatin appears to involve direct neurotoxicity to peripheral nerves. Morphological changes were observed in dorsal root ganglia (DRGs) in rats treated with oxaliplatin [6]. Because chronic neurotoxicity is a serious doselimiting side effect, patients decline oxaliplatin treatment [7]. Chronic pain often lasts for a long time, resulting in poor quality of life for affected individuals.

Oxaliplatin is degraded to a variety of reactive intermediates, including oxalate and dichloro (1, 2-diaminocyclohexane) platinum [8]. In rats, repeated administration of oxaliplatin causes cold hyperalgesia in the acute phase and mechanical allodynia in the chronic phase [9]. Oxalate derived from oxaliplatin is implicated in cold hyperalgesia but not mechanical allodynia [9]. Chronic neurotoxicity is difficult to treat. Therefore, use of analgesics in the clinical management of chronic neurotoxicity is important.

Various drugs have been developed to prevent or treat these two types of oxaliplatin-induced peripheral neurotoxicity. Gamelin et al. reported that calcium and magnesium infusions relieve peripheral neurotoxicity [10]. Several antiepileptic agents, such as carbamazepine and gabapentin [11], amifostine [12], and glutathione [13] have demonstrated some degree of success in preventing and treating oxaliplatin-induced acute neurotoxicity. However, no standard treatment for neurotoxicity has been developed.

Neurotropin, a non-protein extract derived from inflamed rabbit skin following inoculation with vaccinia virus, is an analgesic agent. Neurotropin has been used clinically for many years in Japan for the treatment of various types of chronic pain, including post-herpetic neuralgia, subacute myelo-optic neuropathy, lower back pain, and other neuropathies. The mechanism of action of neurotropin has been partially clarified. No experimental study, however, has been undertaken to assess oxaliplatininduced neurotoxicity using quantitative in vitro high-throughput image analysis. In the present study, to elucidate the action of neurotropin on DRG neurons, we explored the antioxidative and anti-inflammatory activity of neurotropin. We also examined the effects of neurotropin on neurotoxicity in rat DRG neurons using the ImageXpress screening system at $10 \times$ magnification. This screening system for evaluating neurotoxicity would benefit from automated and quantitative in vitro assays that observe morphological changes in DRG neurons.

We hypothesized that oxaliplatin causes neuronal synapses to deteriorate in the early phase, finally causing neurite degeneration and death of neurons. Thus, we used the ImageXpress screening system to assess synapse plasticity and changes in neuronal morphology to assess putative neuroprotective effects of neurotropin. Thus, we used the ImageXpress screening assay to quantify the morphological changes in neurites and cell bodies. After image acquisition, the images were auto-quantified by specialized image analysis software, MetaXpress Version 1.7. The analysis data are potential indicators of neurotoxicity, and we were able to quantify changes in the morphology of these neurons under different conditions.

Here, we report a new way of quantifying morphological changes in cultured rat DRG neurons treated with oxaliplatin and neurotropin utilizing this automated, quantitative, highthroughput imaging system. Using this assay system, we were able to quantify cell and neurite parameters.

The main contributions of this paper are: (1) we found significant increases in neurite length, neurite area, cell body area, and neurite branching in the presence of neurotropin in DRG neurons treated with oxaliplatin. (2) We demonstrated that neurotropin reversed oxaliplatin-induced apoptosis via suppression of the p38 mitogen-activated protein kinase signaling pathway.

\section{Related Work}

Kawashiri et al. showed that repeated administration of neurotropin can alleviate oxaliplatin-induced mechanical allodynia by suppressing axonal degeneration [14]. A prospective, open-label, randomized, single-center, pilot clinical trial to evaluate treatments using neurotropin for patients with oxaliplatin-induced neurotoxicity reported that oral administration of neurotropin may effectively and safely decrease chronic neurotoxicity [15].

\section{Materials and Methods}

\section{Primary culture of adult DRG neurons}

All animal experimental protocols were approved by the Institutional Animal Care and Use Committee of Ehime University, and the experiments were conducted according to the Ethical Guidelines for Animal Experiments of Ehime University. Sixweek-old female Sprague-Dawley rats (Charles River, Yokohama, Japan) were housed individually and maintained under light/ dark cycles, with food and water available ad libitum. After 1 week of acclimation, the rats were anesthetized with isoflurane and euthanized. Primary cultures of adult DRG neurons were established as previously described [16]. Briefly, ganglia from the thoracolumbar region were dissected out from the rats and digested in Ham's F-12 medium (Wako, Osaka, Japan) containing $2 \mathrm{mg} / \mathrm{mL}$ collagenase (Worthington Biochemical Corporation, Freehood, NJ) at $37^{\circ} \mathrm{C}$ for $120 \mathrm{~min}$. Subsequently, the ganglia were incubated in $\mathrm{Ca}^{2+} / \mathrm{Mg}^{2+}$-free Hanks' balanced salt solution (HBSS; Sigma-Aldrich, St. Louis, MO) containing $2.5 \mathrm{mg} / \mathrm{mL}$ trypsin (Sigma-Aldrich) at $37^{\circ} \mathrm{C}$ for $15 \mathrm{~min}$. Ganglia were then triturated using a fire-polished glass pipette in HBSS and purified by density gradient centrifugation ( $5 \mathrm{~min}, 200 \times \mathrm{g}$ ) with $30 \%$ Percoll (GE Healthcare Bio-Science Corp., Piscataway, NJ). The isolated cells were plated at a density of 5000 cells/well in 96-well 
plates coated with poly-L-lysine (Sigma-Aldrich). DRG neurons were cultured for 2 weeks in Ham's F-12 medium containing $10 \%$ fetal bovine serum (Hyclone, Logan, UT), 2\% B-27 serumfree supplement (Thermo Fisher Scientific, Rockford, IL), and 1\% penicillin-streptomycin-amphotericin B suspension (Wako) at $37^{\circ} \mathrm{C}$ in a humidified $5 \% \mathrm{CO}_{2}$ atmosphere.

\section{Drugs and cell culture}

Neurotropin was a kind gift from Nippon Zoki Pharmaceutical Co., Ltd. Neurotropin was dissolved in Ham's F-12 medium. Oxaliplatin was purchased from Tokyo Chemical Industry Co. Ltd. DRG neurons were treated with neurotropin at concentrations of 4 and 40 neurotropin units (NU) $\mathrm{mNU} / \mathrm{mL}$ and/or $0.1 \mu \mathrm{M}$ oxaliplatin.

\section{Fluorescent staining}

Cells were fixed with $4 \%$ paraformaldehyde in phosphatebuffered saline (PBS) at $4^{\circ} \mathrm{C}$ for $15 \mathrm{~min}$, rinsed twice with PBS, and then blocked with BlockHen (Aves Labs, Tigard, OR) for 15 $\mathrm{min}$ at room temperature to reduce nonspecific staining. The 96-well plates were incubated with anti-beta III tubulin antibody (1:1000; Abcam, Cambridge, UK) as the primary antibody at room temperature for 1 hour and rinsed three times. The 96well plates were incubated with goat anti-chicken IgY H\&L (FITC; 1:500; Abcam) as the secondary antibody at room temperature for 1 hour, rinsed three times, and then stained with Hoechst 33342 (0.1 mg/mL; Dojindo, Kumamoto, Japan) for $20 \mathrm{~min}$ at room temperature.

\section{Analysis with ImageXpress and MetaXpress}

The cells were examined with a fluorescence microscope, ImageXpress Micro (Molecular Devices, Sunnyvale, CA). The images were analyzed with high content image processing software, MetaXpress (Molecular Devices). The images were acquired with a $600 \mathrm{~ms}$ exposure time. Neurites and cell bodies were identified using the Angiogenesis module of MetaXpress. Neurite parameters were set to a 2-pixel minimum and a 22-pixel maximum, and cell body parameters were set to a width of over 22 pixels. Total neurite length, total neurite area, total cell body area, and total neurite branching per well were determined using the ImageXpress screening system. The Angiogenesis module has low time complexity and is suitable for this analysis. The algorithm flowchart is shown in Figure 1.

\section{Cytotoxicity of neurotropin}

The toxicity of neurotropin was assayed using the direct cell counting function of the ImageXpress software. Two weeks after seeding the DRG neurons, different concentrations of neurotropin were added to the 96-well plate. The cultures were further incubated for 1 week, and then neuronal nuclei were stained with Hoechst $33342(0.1 \mathrm{mg} / \mathrm{mL})$ for $20 \mathrm{~min}$ at room temperature. Six replicates of all experiments were performed. The relative cell viability (\%) was expressed as the percent of untreated control cells in cell culture medium without neurotropin. The cell counting algorithm, which is the cell scoring application module of MetaXpress software, detected stained nuclei in each well of the 96-well plate.

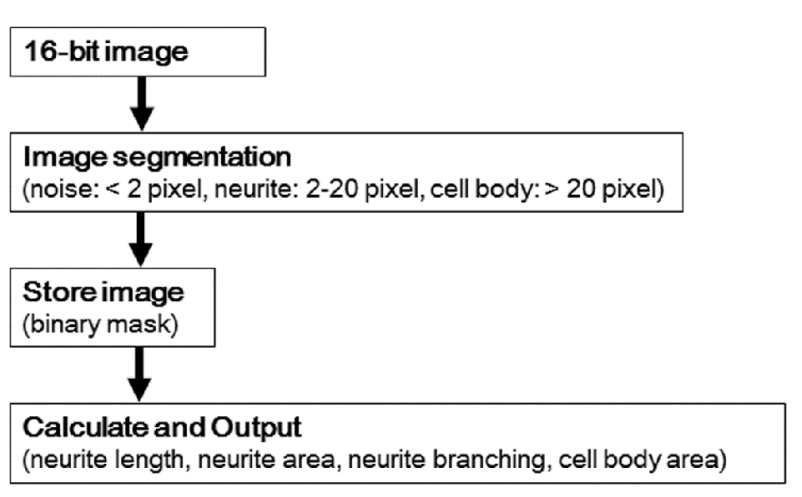

Figure 1 Algorithm flowchart of Angiogenesis module.

\section{Western blot}

Cells treated with neurotropin and/or oxaliplatin were lysed in Pierce RIPA Buffer (Pierce Biotechnology, Rockford, IL) and subjected to western blot analysis. Each sample containing 30 $\mu \mathrm{g}$ of protein was electrophoresed on a $10 \%$ SDS gel at $200 \mathrm{~V}$ for $60 \mathrm{~min}$ at room temperature. Proteins were transferred onto polyvinylidene difluoride membranes (Bio-Rad, Hercules, and $(\mathrm{A})$. Blots were blocked in PBS with $5 \%$ nonfat dry milk and $0.1 \%$ Tween for 1 hour at room temperature. Membranes were washed and then incubated with 1:1000 dilutions of monoclonal thioredoxin (TRX) rabbit antibody (Cell Signaling Technology, Beverly, MA), monoclonal p38 mitogen-activated protein kinase (MAPK) rabbit antibody (Cell Signaling Technology), monoclonal phospho-p38 (pp38) MAPK rabbit antibody (Cell Signaling Technology), c-Jun N-terminal kinase (JNK) rabbit antibody (Cell Signaling Technology), phospho-JNK rabbit antibody (Cell Signaling Technology), or beta-actin rabbit antibody (Cell Signaling Technology) at $4^{\circ} \mathrm{C}$ overnight. Next, the membranes were incubated with anti-rabbit IgG HRP conjugate (1:2000; Promega, Madison, WI) as the secondary antibody at room temperature for 1 hour. Bands were visualized by chemiluminescence using ImmunoStar LD (Wako), and luminescent images were analyzed with ImageQuant LAS 4010 (GE Healthcare, Chicago, IL).

\section{Statistical Analysis}

Data were analyzed using a repeated measures one-way analysis of variance followed by Tukey's test for post-hoc comparisons. A $p$ value less than 0.05 denoted the presence of a statistically significant difference. All statistical analyses were performed using Graphpad Prism version 6.0 (Graphpad Software, San Diego, CA).

\section{Results}

\section{The viability of DRG neurons exposed to neu- rotropin}

The viability of DRG neurons exposed to neurotropin is shown in Figure 2A. We found no change in the viability of DRG neurons treated with different concentrations ( 4 and $40 \mathrm{mNU} / \mathrm{mL}$ ) of neurotropin. The general morphology of the DRG neurons 
incubated with or without neurotropin in phase contrast microscopy is shown in Figure 2B-2D. No significant cytotoxicity at concentrations below $40 \mathrm{mNU} / \mathrm{mL}$ was observed.

The effects of neurotropin on neurite length, neurite area, cell body area, and neurite branching

Because neurotropin may exert a protective effect against oxaliplatin-induced mechanical allodynia by suppressing axonal degeneration [14], we developed an automated protocol to determine whether neurotropin protects against oxaliplatininduced morphological changes in DRG neurons. DRG neurons were grown in the absence of neurotropin, $4 \mathrm{mNU} / \mathrm{mL}$ neurotropin, or $40 \mathrm{mNU} / \mathrm{mL}$ neurotropin and treated with 0.1 $\mu \mathrm{M}$ oxaliplatin for 7 days and fixed. Representative images of vehicle and oxaliplatin-treated cultures in the absence of added neurotropin and in the presence of 4 or $40 \mathrm{mNU} / \mathrm{mL}$ neurotropin at day 7 are shown in Figure 3. Quantification of neurite length,
A

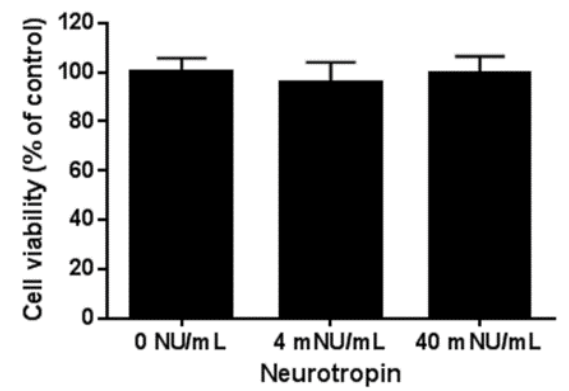

B

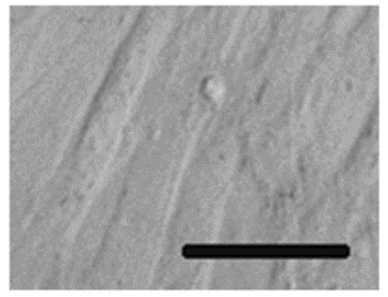

C



D

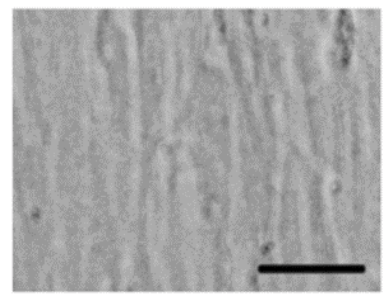

Figure 2 Cytotoxicity of neurotropin in DRG neurons. (A) Cell viability of DRG neurons cultured in the absence or presence of neurotropin (4 or $40 \mathrm{mNU} / \mathrm{mL}$ ) for 1 week. The general morphology of DRG neurons incubated without neurotropin (B). The general morphology of DRG neurons incubated with $4 \mathrm{mNU} / \mathrm{mL}$ (C) or $40 \mathrm{mNU} / \mathrm{mL}$ (D) neurotropin. Scale bar: $40 \mu \mathrm{m}$. (original magnification, $\times 200$ ). Abbreviations: DRG, dorsal root ganglion; NU, neurotropin units.

\section{A}
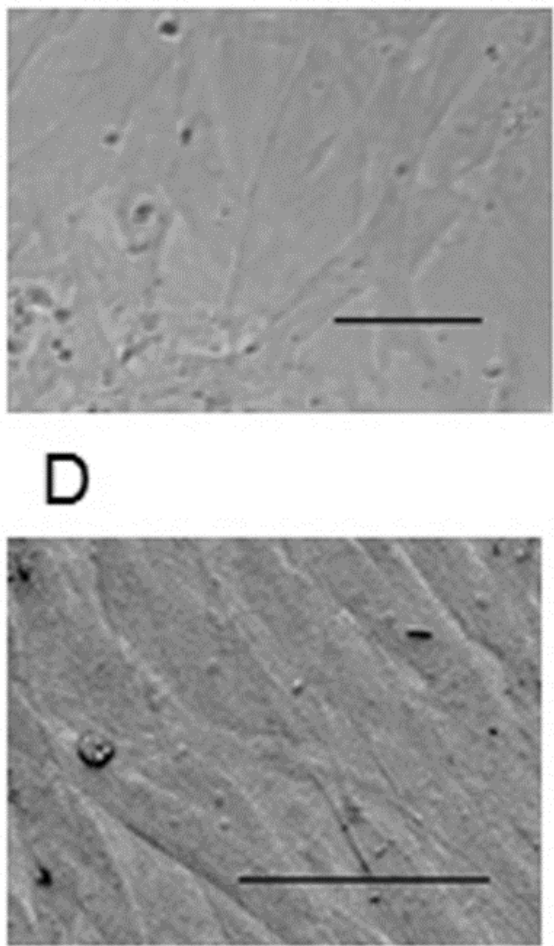

B
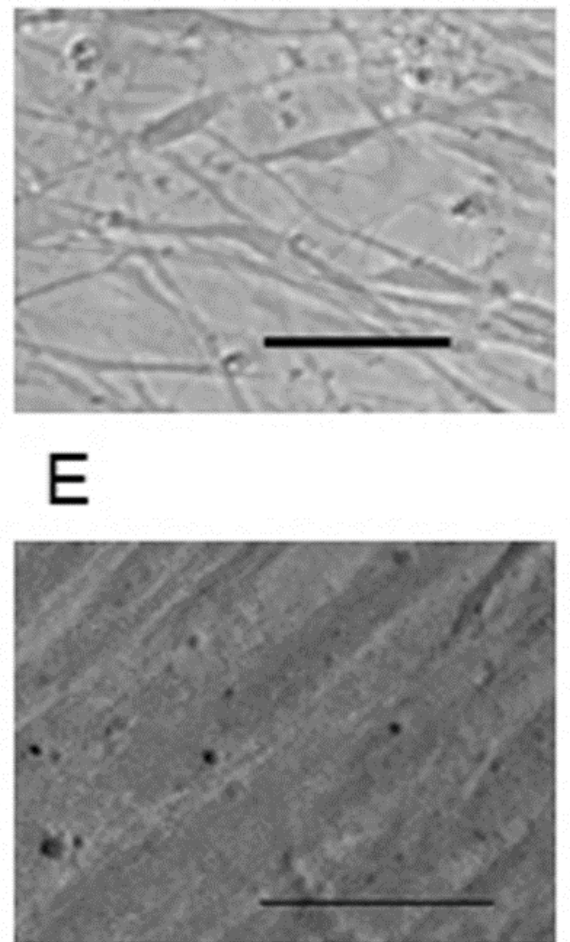
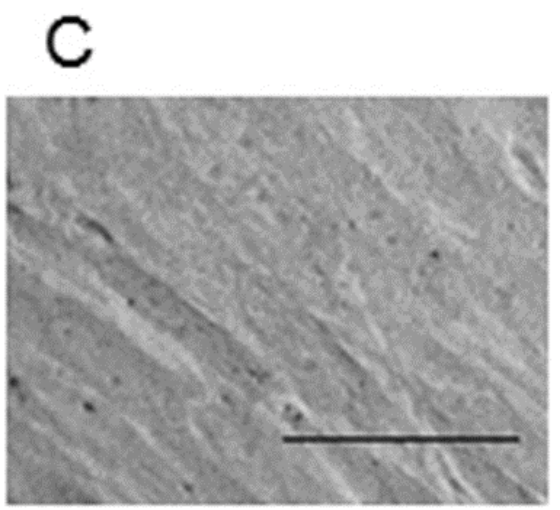

F

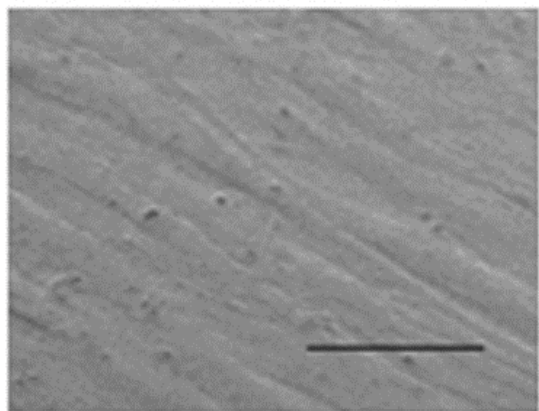

Figure 3 Representative images of vehicle- and oxaliplatin-treated cultures in the absence of added neurotropin and in the presence of 4 or $40 \mathrm{mNU} / \mathrm{mL}$ neurotropin at day 7. (A) Control. (B) Oxaliplatin $(0.1 \mu \mathrm{M})$. (C) Neurotropin $(4 \mathrm{mNU} / \mathrm{mL})$. (D) Neurotropin (40 $\mathrm{mNU} / \mathrm{mL})$. (E) Oxaliplatin $(0.1 \mu \mathrm{M})$ plus neurotropin $(4 \mathrm{mNU} / \mathrm{mL})$. (F) Oxaliplatin $(0.1 \mu \mathrm{M})$ plus neurotropin $(40 \mathrm{mNU} / \mathrm{mL})$. Scale bar: $40 \mu \mathrm{m}$, Abbreviation: NU, neurotropin units. 
neurite area, cell body area, and neurite branching in DRG neurons treated with control or oxaliplatin in the presence or absence of neurotropin was performed using the ImageXpress screening system (Figure 4). The results also indicated significant effects of neurotropin on neurite length, neurite area, cell body area, and neurite branching in DRG neurons treated with oxaliplatin.

\section{Activation pp38 and JNK in DRG neurons}

Western blotting of DRG neurons demonstrated activation of the p38 MAPK pathway (Figure 5). Exposure of DRG neurons to neurotropin $(40 \mathrm{mNU} / \mathrm{mL})$ significantly decreased the level of pp38, whereas the level of p38 was unchanged. In contrast, the JNK MAPK pathway was not activated (Figure 5).

\section{Expression of TRX in DRG neurons}

We examined whether TRX expression was different between oxaliplatin- and neurotropin-treated DRG neurons and DRG neurons treated with oxaliplatin only. Western blotting showed that neurotropin induced the expression of TRX in oxaliplatintreated DRG neurons in a dose-dependent manner (Figure 6).
A

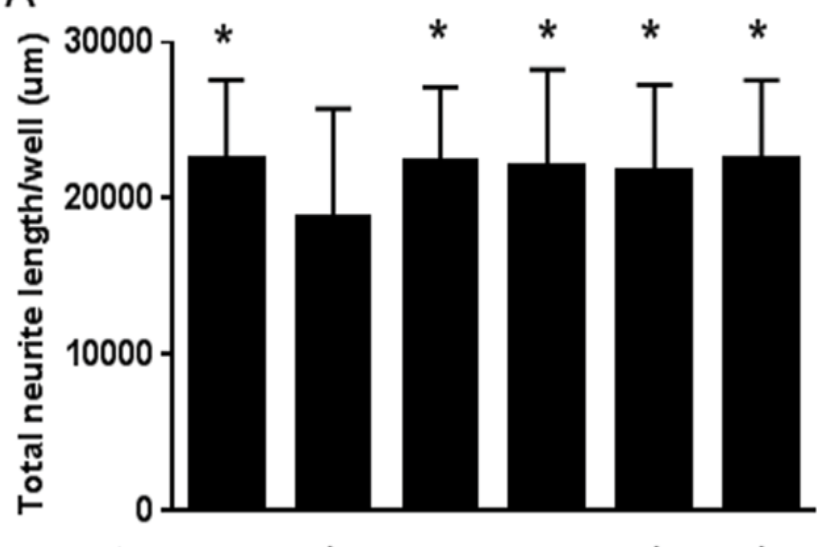

Oxaliplatin $\quad-\quad+\quad+\quad-\quad-\quad+\quad+\quad+$ (0.1uM)

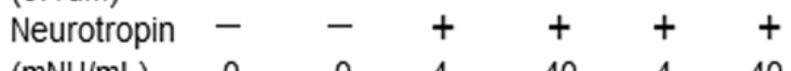
$\begin{array}{lllllll}(\mathrm{mNU} / \mathrm{mL}) & 0 & 0 & 4 & 40 & 4 & 40\end{array}$

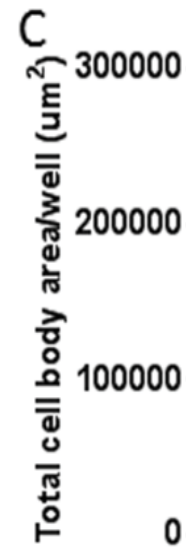

Oxaliplatin (0.1uM) Neurotropin $-\quad-\quad-\quad+\quad+\quad+\quad+\quad+$ $(\mathrm{mNU} / \mathrm{mL})$
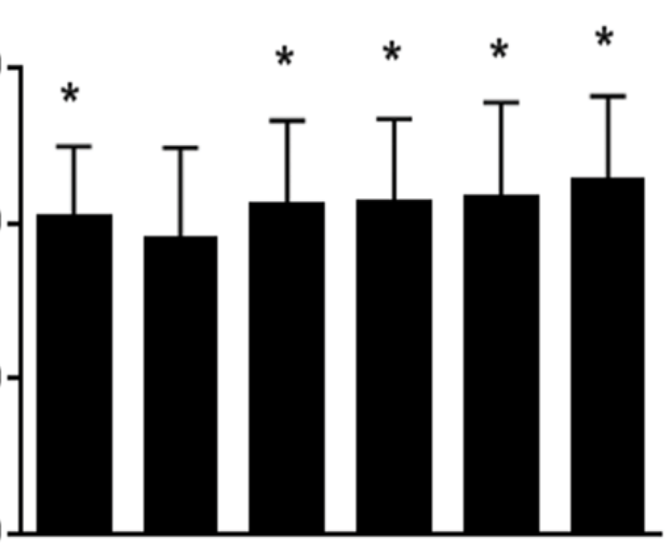

Figure 4

\section{B}

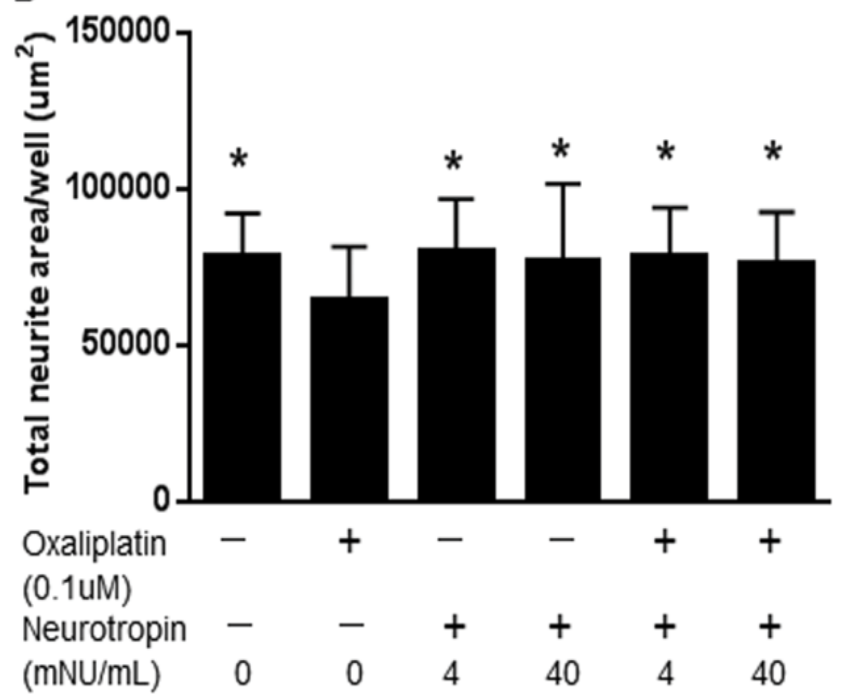

D

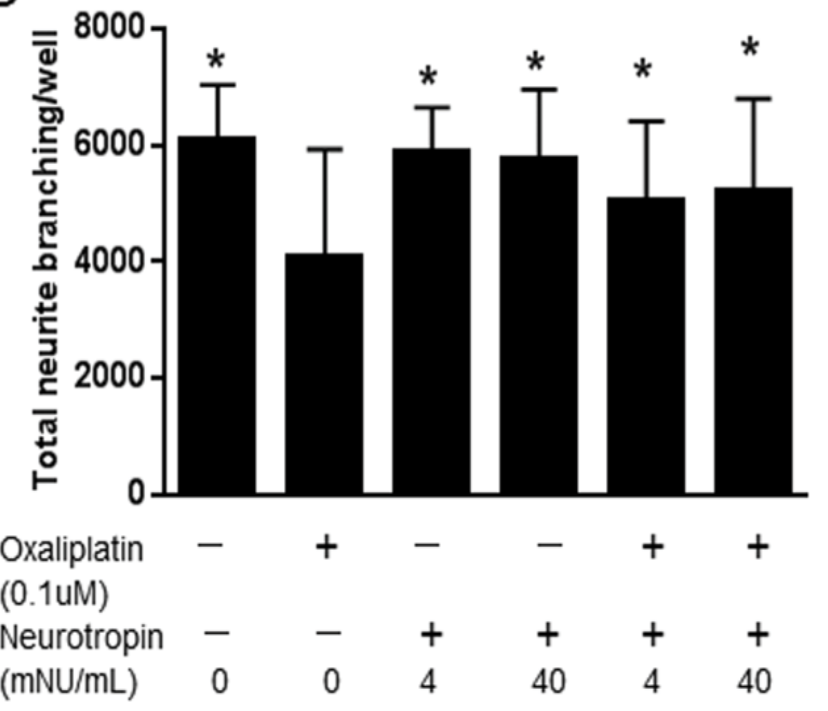
length, total neurite area, total cell body area, and total neurite branching per well were determined using the ImageXpress screening system. ${ }^{*} p<0.05$, significantly different from oxaliplatin-treated DRG neurons in the absence of neurotropin, as determined by one-way ANOVA followed by Tukey's test for post-hoc comparisons. Abbreviations: DRG, dorsal root ganglion; ANOVA, analysis of variance; NU, neurotropin units. 


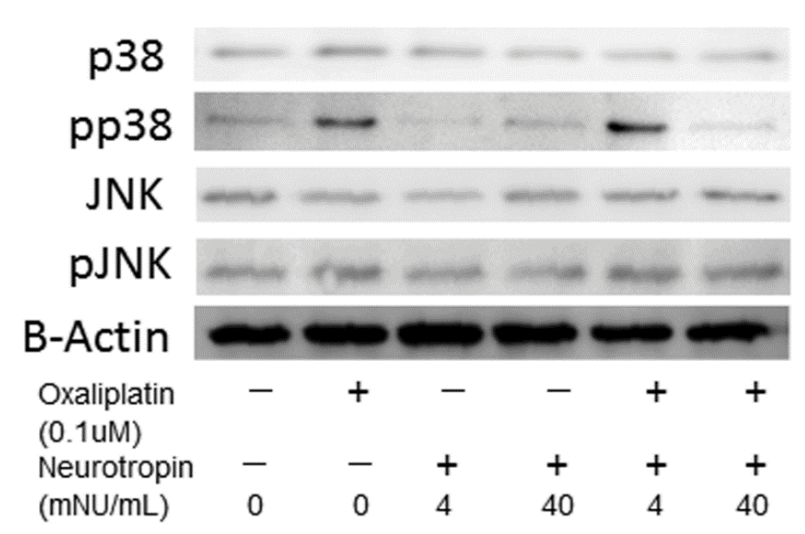

Figure 5 Western blotting image of p38, pp38, JNK, pJNK, and $\beta$-actin expression in DRG neurons. Thebottom panel is a loading control with $\beta$-actin. Oxaliplatintreated neurons in the absence of neurotropin showed much stronger pp38 expression compared with oxaliplatin-treated neurons in the presence of neurotropin (40 mNU/mL). Abbreviations: DRG, dorsal root ganglion; JNK, C-Jun N-terminal kinase; $\mathrm{NU}$, neurotropin units.

\section{TRX B-Actin \\ Oxaliplatin \\ (0.1uM) \\ Neurotropin \\ $(\mathrm{mNU} / \mathrm{mL})$

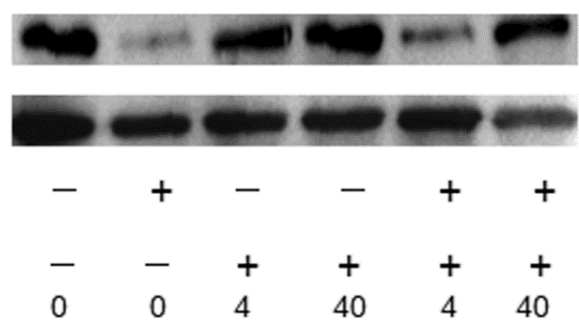

Figure 6 Western blotting image of TRX and $\beta$-actin expression in DRG neurons. The bottom panel is a loading control with $\beta$-actin. Oxaliplatintreated neurons in the absence of neurotropin showed much lower TRX expression compared with oxaliplatin-treated neurons in the presence of neurotropin $(40 \mathrm{mNU} / \mathrm{mL})$. Abbreviations: DRG, dorsal root ganglion; TRX, thioredoxin; NU, neurotropin units.

\section{Discussion}

Neurotropin decreases chronic neurotoxicity, but the mechanism of action of neurotropin has been only partially clarified. Accordingly, in vitro experiments were carried out to elucidate the mechanism of action of neurotropin in DRG neurons.

In this report, we describe an automated, quantitative, highthroughput imaging system for multiparametric measurement of the cellular response to investigate the efficacy of neurotropin. In vitro high-throughput screening assays are needed to examine and quantitate the cytoprotective effects of drugs on DRG neurons. High-throughput imaging of neurons is particularly useful for toxicity screening, as it can measure cell viability and generate multiparametric readouts $[17,18]$.
In this study, we report the establishment of a quantitative highthroughput analysis method that included multiple parameters, allowing for assessment of the morphological features of DRG neurons. Specifically, the cytoprotective effect of neurotropin was characterized by assessing the neurite length, neurite area, cell body area, and neurite branching. We hypothesized that a functional in vitro assay for neuronal morphology would be useful for quantitation of the neuroprotective effect of neurotropin. As shown in Figure 2, no neurotoxicity effect was observed in DRG neurons treated with neurotropin at concentrations of 4 and 40 $\mathrm{mNU} / \mathrm{mL}$. Because neurotropin significantly increased the neurite length, neurite area, cell body area, and neurite branching, neurotropin treatment may decrease the neurotoxicity of oxaliplatin (Figure 4).

Enhanced stability of microtubules leads to an increase in the length of microtubule polymers and subsequently results in an increase in neurite length [19]. Meanwhile, neurite stability could be a reflection of microtubule stability, which may accordingly prevent neurite retraction. Therefore, neurotropin may be neuroprotective due to enhancement of microtubule stability.

Oxaliplatin treatment simultaneously decreases the total RNA content of DRG neurons and alters the DRG neuronal size [20]. Oxaliplatin induces global transcriptional arrest, leading in turn to neuronal atrophy. DRG neurons may be especially vulnerable to oxaliplatin-induced neurotoxicity because they require high levels of global transcriptional activity. The data presented in this paper demonstrated that oxaliplatin reduced the cell body size. In contrast, cell body size was maintained in the presence of neurotropin. These considerations have led us to hypothesize that neurotropin protects DRG neurons from oxaliplatin-induced inhibition of transcription.

The increase in neurite branching after treatment of DRG neurons with neurotropin was unexpected, and the mechanism by which neurotropin increases neurite branching remains unexplained. Cholesterol affects the membrane properties of neurites by improving neurite adhesion to the extracellular matrix and increasing the number of neuronal growth cones, both of which enhance neurite branching [21]. Neurotropin significantly reduces axonal transport in a concentration-dependent manner in DRG neurons [22]. Axonal transport could convey some components related to cholesterol metabolism, resulting in decreased neurite branching of DRG neurons.

Oxaliplatin, which is a platinum-based anticancer drug, causes peripheral neurotoxicity by damaging DRG neurons. This mechanism of action involves platinum binding to DNA bases, which inhibits DNA replication and transcription, resulting in tumor cell cycle arrest and apoptosis [23]. Our results indicated that neurotropin prevented oxaliplatin-induced neurotoxicity and cell death in cultured DRG neurons. MAPK signaling plays an important role in the sensitivity to anticancer drugs [24]. Activated MAPK transmits extracellular signals to regulate many cellular processes such as cell proliferation, migration, differentiation [25], and apoptosis [26]. Six mammalian MAPK signaling pathways have been characterized in detail: extracellular signalregulated kinase-1/2 (ERK1/2), ERK3/4, ERK5, ERK7/8, JNK, and 
the p38 isoforms $\alpha / \beta / \gamma($ ERK6)/ $\delta$ [27-30]. Stress-activated MAPK, p38 MAPK, and JNK play an important role in the treatment outcome and sensitivity to anticancer drugs [24]. Treatment with oxaliplatin activates phosphorylation of p38 MAPK [31] The activation of JNK and p38 MAPK is critical for induction of apoptosis [32]. Western blot analysis was performed to detect JNK, pJNK, p38, and pp38 protein levels in DRG neurons following oxaliplatin treatment (Figure 5). We observed that neurotropin did not affect the phosphorylation of JNK but suppressed the phosphorylation of p38 (Figure 5). This finding suggests that neurotropin may alleviate oxaliplatin-induced apoptosis via suppression of the p38 MAPK signaling pathway.

Although the mechanism underlying oxaliplatin-induced neurotoxicity is not clearly understood, oxaliplatin may generate formation of reactive oxygen species, which could interfere with the antioxidant defense system, causing cytotoxic effects and apoptosis [33-36]. TRX is a multifunctional protein with a molecular mass of $12 \mathrm{kDa}$ that acts as an antioxidant and antiapoptotic molecule [36,37]. TRX is a binding partner of apoptosis signal-regulating kinase 1 (ASK1) and a negative regulator of ASK1 [38]. TRX directly binds to ASK1 and inhibits ASK1 kinase activity [38]. ASK1 is a MAPK kinase kinase (MKK) that activates p38 MAPK (via activation of MKK3/MKK6) [39]. In this study, we also examined the expression of TRX by western blotting (Figure 6). Treatment of neurotropin enhanced the expression of TRX in a concentration-dependent manner (Figure 6), inhibited activation of the p38 pathway (Figure 5), and protected DRG neurons from oxaliplatin-induced neurotoxicity (Figures 3 and 4). These results suggest that neurotropin may alleviate oxaliplatin-induced apoptosis via the ASK1-p38 signaling pathway in DRG neurons through the induction of TRX.

Neurotropin is a well-known analgesic agent, but its neuroprotective effects have not been investigated. Neurotropin enhances cytoprotective effects against a variety of oxidative stressors in lung cells [40]. The effect mediated by the induction of TRX attenuates reactive oxygen species production [40]. The mechanism of pain relief by neurotropin is believed to be inhibition of kallikrein-kinin activity $[41,42]$. Neurotropin contains a variety of non-protein components, which may mediate antioxidative and anti-inflammatory activity. Cytoprotective effects of TRX on oxidative lung injury have been observed $[40,43]$. Taken together with our in vitro data, the induction of TRX after neurotropin treatment may inhibit apoptosis in DRG neurons.

\section{References}

1 Favoriti P, Carbone G, Greco M, Pirozzi F, Pirozzi RE, et al. (2016) Worldwide burden of colorectal cancer: a review. Updates Surg 68: 7-11.

2 Siegel RL, Miller KD, Jemal A (2016) Cancer statistics, CA Cancer J Clin 66: 7-30

3 Punt CJ (2004) New options and old dilemmas in the treatment of patients with advanced colorectal cancer. Ann Oncol 15: 1453-1459.
Our results indicate that the neuroprotective effects of neurotropin were more pronounced at the higher dose (40 $\mathrm{mNU} / \mathrm{mL}$ ) than at the lower dose $(4 \mathrm{mNU} / \mathrm{mL})$, suggesting that a lower dose of neurotropin is sufficient to protect neurites against oxaliplatin-induced neurotoxicity, but not to inhibit oxaliplatin-induced apoptosis. Given the anti-apoptotic effect of neurotropin on DRG neurons, neurotropin may have preventive or therapeutic effects on severe inflammatory diseases.

The results of these in vitro studies may suggest a role for neurotropin as a useful neuroprotective and anti-apoptotic agent. Our in vitro studies revealed significant enhancing effects of neurotropin on neurite properties in DRG neurons, and thus, additional studies are needed to further elucidate roles for neurotropin.

\section{Conclusion}

Significant increases in neurite length, neurite area, cell body area, and neurite branching were observed in DRG neurons in the presence of neurotropin. Neurotropin provided neuroprotective effects against oxaliplatin-induced neurotoxicity. The results of these in vitro studies suggest a possible neuroprotective and anti-apoptotic role for neurotropin. Further studies are needed to elucidate roles for neurotropin in DRG neurons.

\section{Acknowledgments}

We thank Nippon Zoki Pharmaceutical Company for the generous gift of neurotropin. The authors also thank Takeshi Kiyoi at the Division of Analytical Bio-Medicine, the Advanced Research Support Center (ADRES), Ehime University for his technical assistance.

\section{Author Contributions}

SA performed the experiments and collected and analyzed the data. MS conceived and designed the experiments, participated in the data collection process, analyzed the data and wrote the manuscript. MY and YW approved the proposal with some revisions and reviewed the manuscript. All authors revised the paper critically and agreed to be accountable for the work.

\section{Conflict of Interest}

The authors report no conflicts of interest in this work. The authors received no financial support for this study. All relevant data are included in this paper.

4 Dault R, Rousseau MP, Beaudoin A, Frenette MA, Lemay F, et al. (2016) Impact of oxaliplatin-induced neuropathy in patients with colorectal cancer: a prospective evaluation at a single institution. Curr Oncol 23: e65-69.

5 Wilson RH, Lehky T, Thomas RR, Quinn MG, Floeter MK, et al. (2002) Acute oxaliplatin-induced peripheral nerve hyperexcitability. J Clin oncol 20: 1767-1774.

6 Saif MW, Reardon J (2005) Management of oxaliplatin-induced peripheral neuropathy. Ther Clin Risk Manag 1: 249-258. 
7 Park SB, Lin CS, Krishnan AV, Goldstein D, Friedlander ML, et al. (2011) Long-term neuropathy after oxaliplatin treatment: challenging the dictum of reversibility. Oncologist 16: 708-716.

8 Graham MA, Lockwood GF, Greenslade D, Brienza S, Bayssas M, et al. (2000) Clinical pharmacokinetics of oxaliplatin: a critical review. Clin cancer Res 6: 1205-1218.

9 Sakurai M, Egashira N, Kawashiri T, Yano T, Ikesue H, et al. (2008) Oxaliplatin-induced neuropathy in the rat: involvement of oxalate in cold hyperalgesia but not mechanical allodynia. Pain 147: 165-174.

10 Gamelin L, Boisdron-Celle M, Delva R, Guérin-Meyer V, Ifrah N, et al. (2004) Prevention of oxaliplatin-related neurotoxicity by calcium and magnesium infusions: a retrospective study of 161 patients receiving oxaliplatin combined with 5-Fluorouracil and leucovorin for advanced colorectal cancer. Clin Cancer Res 10: 4055-4061.

11 Lersch C, Schmelz R, Eckel F, Erdmann J, Mayr M, et al. (2002) Prevention of oxaliplatin-induced peripheral sensory neuropathy by carbamazepine in patients with advanced colorectal cancer. Clin Colorectal Cancer 2: 54-58.

12 Penz M, Kornek GV, Raderer M, Ulrich-Pur H, Fiebiger W, et al. (2001) Subcutaneous administration of amifostine: a promising therapeutic option in patients with oxaliplatin-related peripheral sensitive neuropathy. Ann Oncol 12: 421-422.

13 Cascinu S, Catalano V, Cordella L, Labianca R, Giordani P, et al. (2002) Neuroprotective effect of reduced glutathione on oxaliplatin-based chemotherapy in advanced colorectal cancer: a randomized, doubleblind, placebo-controlled trial. J Clin Oncol 20: 3478-3483.

14 Kawashiri T, Egashira N, Watanabe H, Ikegami Y, Hirakawa S, et al. (2011) Prevention of oxaliplatin-induced mechanical allodynia and neurodegeneration by neurotropin in the rat model. Eur J Pain 15: 344-350.

15 Zhang RX, Lu ZH, Wan DS, Wu XJ, Ding PR, et al. (2012) Neuroprotective effect of neurotropin on chronic oxaliplatin-induced neurotoxicity in stage II and stage III colorectal cancer patients: results from a prospective, randomised, single-centre, pilot clinical trial. Int $J$ Colorectal Dis 27: 1645-1650.

16 Sango K (2003) Phosphacan and neurocan are repulsive substrata for adhesion and neurite extension of adult rat dorsal root ganglion neurons in vitro. Exp Neurol 182: 1-11.

17 Breier JM, Radio NM, Mundy WR, Shafer TJ (2008) Development of a high-throughput screening assay for chemical effects on proliferation and viability of immortalized human neural progenitor cells. Toxicol Sci 105: 119-133.

18 McNeish J, Roach M, Hambor J, Mather R, Weibley L, et al. (2010) High-throughput screening in embryonic stem cell-derived neurons identifies potentiators of alpha-amino-3-hydroxyl-5-methyl-4 isoxazolepropionate-type glutamate receptors. J Biol Chem 285 17209-17217.

19 Yu W, Schwei MJ, Baas PW (1996) Microtubule transport and assembly during axon growth. J Cell Biol 133: 151-157.

20 Yan F, Liu JJ, Ip V, Jamieson SM, McKeage MJ (2015) Role of platinum DNA damage-induced transcriptional inhibition in chemotherapyinduced neuronal atrophy and peripheral neurotoxicity. J Neurochem 135: 1099-1112.

21 Handelmann GE, Boyles JK, Weisgraber KH, Mahley RW, Pitas RE (1992) Effects of apolipoprotein $\mathrm{E}$, beta-very low density lipoproteins, and cholesterol on the extension of neurites by rabbit dorsal root ganglion neurons in vitro. J Lipid Res 33: 1677-1688.
22 Isonaka R, Takenami T, Katakura T, Kawakami T (2013) Neurotropin inhibits axonal transport in cultured mouse dorsal root ganglion neurons. Neurosci Lett 543: 101-104.

23 Wang D, Lippard SJ (2005) Cellular processing of platinum anticancer drugs. Nat Rev Drug Discov 4: 307-320.

24 Sui X, Kong N, Ye L, Han W, Zhou J, et al. (2014) p38 and JNK MAPK pathways control the balance of apoptosis and autophagy in response to chemotherapeutic agents. Cancer Lett 344: 174-179.

25 Lam E, Kilani RT, Li Y, Tredget EE, Ghahary A (2005) Stratifin-induced matrix metalloproteinase- 1 in fibroblast is mediated by c-fos and p38 mitogen-activated protein kinase activation. J Invest Dermatol 125: 230-238.

26 Wada T, Penninger JM (2004) Mitogen-activated protein kinases in apoptosis regulation. Oncogene 23: 2838-2849.

27 Westermarck J, Kahari VM (1993) Regulation of matrix metalloproteinase expression in tumor invasion. FASEB J 13: 781-792.

28 Schaeffer HJ, Weber MJ (1999) Mitogen-activated protein kinases: specific messages from ubiquitous messengers. Mol Cell Biol 19: 2435-2444.

29 Keyse SM (2000) Protein phosphatases and the regulation of mitogen-activated protein kinase signalling. Curr Opin Cell Biol 12: 186-192.

30 Kyriakis JM, Avruch J (2012) Mammalian MAPK signal transduction pathways activated by stress and inflammation: a 10-year update. Physiol Rev 92: 689-737.

31 Chiu SJ, Chao JI, Lee YJ, Hsu TS (2008) Regulation of gamma-H2AX and securin contribute to apoptosis by oxaliplatin via a p38 mitogenactivated protein kinase-dependent pathway in human colorectal cancer cells. Toxicol Lett 179: 63-70.

32 Xia Z, Dickens M, Raingeaud J, Davis RJ, Greenberg ME (1995) Opposing effects of ERK and JNK-p38 MAP kinases on apoptosis. Science 270: 1326-1331.

33 Santoro V, Jia R, Thompson H, Nijhuis A, Jeffery R, et al. (2016) Role of Reactive Oxygen Species in the Abrogation of Oxaliplatin Activity by Cetuximab in Colorectal Cancer. J Natl Cancer Inst 108: djv394.

34 Di Cesare Mannelli L, Zanardelli M, Failli P, Ghelardini C (2012) Oxaliplatin-induced neuropathy: oxidative stress as pathological mechanism. Protective effect of silibinin. J Pain 13: 276-284.

35 Massicot F, Hache G, David L, Chen D, Leuxe C, et al. (2013) P2X7 Cell Death Receptor Activation and Mitochondrial Impairment in Oxaliplatin-Induced Apoptosis and Neuronal Injury: Cellular Mechanisms and In Vivo Approach. PLoS One 8: e66830.

36 Spector A, Yan GZ, Huang RR, McDermott MJ, Gascoyne PR, et al. (1988) The effect of $\mathrm{H} 2 \mathrm{O} 2$ upon thioredoxin-enriched lens epithelial cells. J Biol Chem 263: 4984-4990.

37 Iwata S, Hori T, Sato N, Hirota K, Sanada T, et al. (1997) Adult T cell leukemia (ATL)-derived factor/human thioredoxin prevents apoptosis of lymphoid cells induced by L-cystine and glutathione depletion: possible involvement of thiol-mediated redox regulation in apoptosis caused by pro-oxidant state. J Immunol 158: 3108-3117.

38 Saitoh M, Nishitoh H, Fujii M, Takeda K, Tobiume K, et al. (1998) Mammalian thioredoxin is a direct inhibitor of apoptosis signalregulating kinase (ASK) 1. EMBO J 17: 2596-2606.

39 Ichijo H, Nishida E, Irie K, Dijke TP, Saitoh M, et al. (1997) Induction of apoptosis by ASK1, a mammalian MAPKKK that activates SAPK/JNK and p38 signaling pathways. Science 275 : 90-94. 
40 Hoshino Y, Nakamura T, Sato A, Mishima M, Yodoi J, et al. (2007) Neurotropin demonstrates cytoprotective effects in lung cells through the induction of thioredoxin-1. Am J Respir Cell Mol Biol 37: 438-446.

41 Ohara H, Namimatsu A, Fukuhara K, Yago H, Yoneda R, et al. (1988) Release of inflammatory mediators by noxious stimuli; effect of neurotropin on the release. Eur J Pharmacol 157: 93-99.
42 Hata T, Kita T, Itoh E, Oyama R, Kawabata A (1988) Mechanism of the analgesic effect of neurotropin. Jpn J Pharmacol 48: 165-173.

43 Ueda S, Nakamura T, Yamada A, Teratani A, Matsui N, et al. (2006) Recombinant human thioredoxin suppresses lipopolysaccharideinduced bronchoalveolar neutrophil infiltration in rat. Life Sci 79: 1170-1177. 